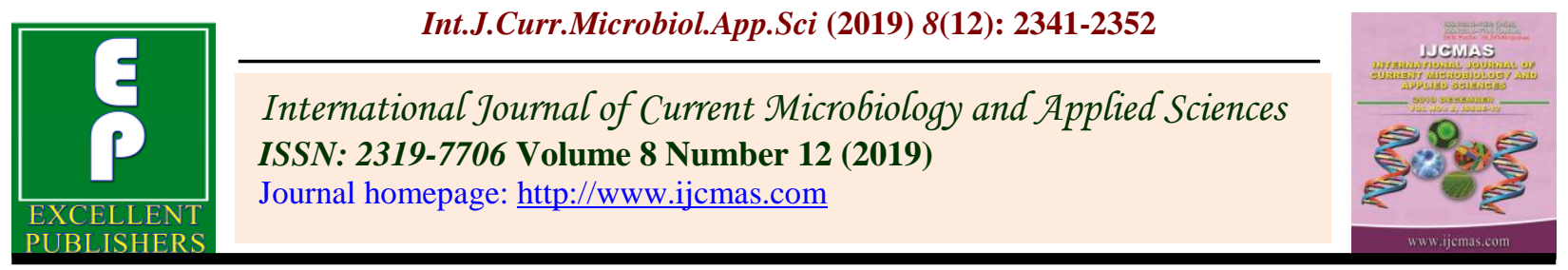

\title{
Genetic Variability, Correlation and Path Coefficient Analysis in Chickpea (Cicer arietinum L.) for Yield and its Component Traits
}

\author{
Sanjay Kumar $^{1 *}$, B. G. Suresh ${ }^{1}$, Anand Kumar ${ }^{2}$ and G. R. Lavanya ${ }^{1}$ \\ ${ }^{1}$ Department of Genetics and Plant Breeding, Sam Higginbottom University of Agriculture, \\ Technology and Sciences, Allahabad, U.P.-211007, India \\ ${ }^{2}$ Bihar Agricultural University, Sabour (Bhagalpur)-813210, India \\ *Corresponding author
}

\begin{tabular}{|l|}
\hline Ke y w or d s \\
Cicer arietinum L., \\
Genetic variability, \\
Correlation, Path \\
analysis and grain \\
yield \\
\hline Article Info \\
\hline Accepted: \\
17 November 2019 \\
Available Online: \\
10 December 2019 \\
\hline
\end{tabular}

A B S T R A C T

The present experiment was carried out at field experimentation centre site of the Genetics and Plant Breeding, Naini Agricultural Institute, Sam Higginbottom University of Agriculture, Technology and Sciences, Allahabad to study about genetic variability, correlation and path analysis in fifty chickpea germplasm during Rabi, 2017-18. The analysis of variance revealed significant differences among the genotypes for all the characters under the study, indicating a high degree of variability present in the material. The values of PCV were found greater than GCV for all the traits studied indicating environmental factors influencing the characters. High PCV and GCV were observed for effective pods per plant $(36.41 \%$ and $34.38 \%)$ followed by total number of pods per plant $(34.85 \%$ and $32.70 \%)$ and seed index $(29.98 \%$ and $29.78 \%$ ) indicated large extent of genetic variability for these traits in the material. High heritability were recorded by seed index $(98.61 \%)$ followed by harvest index (90.81\%), effective pods per plant $(89.14 \%)$, total number of pods per plant $(88.01 \%)$, secondary branches per plant $(84.42 \%)$, primary branches per plant $(84.31 \%)$ and plant height $(81.03 \%)$.High heritability along with high genetic advance was observed for the traits, viz., effective pods per plant, total number of pods per plant, seed index, primary branches per plant and secondary branches per plant indicated that the characters were mostly governed by additive gene effects. So direct selection of these characters based on phenotypic expression by simple selection method would be effective due to accumulation of more additive genes leading to further improvement. Grain yield per plant showed positive significant genotypic and phenotypic correlations with biological yield/plant, seed index, harvest index, effective pod/plant, total number of pods/plant and secondary branches per plant whereas significant negative correlations with days to $50 \%$ flowering respectively. At the both phenotypic and genotypic levels, biological yield per plant, harvest index, days to maturity, chlorophyll index and plant height had direct positive effect on grain yield per plant had given the highest contribution on yield per plant. So the utmost importance should be given to these characters during the selection for yield improvement in chickpea. 


\section{Introduction}

Chickpea (Cicer arietinum L.) is a self pollinated crop, with $2 \mathrm{n}=2 \mathrm{x}=16$ chromosomes and genome size of $732 \mathrm{Mb}$. India contributes major share of world's chickpea area $(70 \%)$ and production $(67 \%)$ and continues to be the largest chickpea producing nation. In India chickpea is cultivated mostly in as a rainfed crop $(68 \%$ area) in all parts of the country (Dixit et al., 2019). During 2017-18, chickpea was cultivated in an area of $10.76 \mathrm{~m}$ ha with production of 11.20 million tons and productivity of $1032 \mathrm{~kg} / \mathrm{ha}$. The improvement in crop yield depends upon the magnitude of genetic variability available in breeding material and the extent to which the yield component traits are heritable from generation to generation. The genetic variability can thus be a choice for selecting suitable parents; however, the quantitative characters are prone for environmental influence that necessitates the partitioning of overall variances as heritable and non-heritable components for efficient breeding programme (Hamdi, 1992). Absolute variability in different characters cannot be the decisive factor for deciding as to which character is showing the highest degree of variability. The relative values of phenotypic and genotypic coefficient of variation, therefore gives an idea about the magnitude of variability present in a population since the estimate of genotypic and phenotypic coefficient of variation, heritability and expected genetic advance are useful for yield improvement and the above values were estimated to know the scope of improvement in the yield of chickpea genotypes. Yield is a complex character and influenced by many environmental factors, direct selection based on yield may not be rewarding. Therefore a basic understanding of the nature and magnitude of correlation among component traits towards yield is essential. Correlation coefficient and path analysis offers a means of determining the important traits influencing the dependent trait such as seed yield and it also helps in the determination of the selection criteria for simultaneous improvement of various characters along with economic yield. Therefore the present study was undertaken to assess the genetic variability, correlation and path analysis for yield and yield components traits.

\section{Materials and Methods}

The experimental materials comprised of fifty germplasm of chickpea were sown on $6^{\text {th }}$ November, 2017 at department of Genetics and Plant Breeding, SHUATS, Prayagraj. The experiment was laid in randomized complete block design with three replications. The plot size was $4.8 \mathrm{~m}^{2}$ with each row of $4.0 \mathrm{~m}$ length. Interrow spacing was kept $30 \mathrm{~cm}$ and plant to plant spacing was $10 \mathrm{~cm}$. The recommended packages and practices were followed for raise a healthy crop. Data for 14 quantitative traits were recorded viz. days to $50 \%$ flowering (D50F), days to maturity (DM), chlorophyll index (CHL. Index), Canopy temperature at vegetative stage (CTVS), Canopy temperature at pod filling stage (CTPFS), plant height $(\mathrm{PH})$, number of primary branches per plant $(\mathrm{PB})$, number of secondary branches per plant (SB), number of pods per plant (TPPP), effective pods per plant (EPPP), biological yield per plant (BY), harvest index (HI), seed index (SI) and seed yield per plant (GYPP).

The days to $50 \%$ flowering and days to maturity were accounted on a plot basis and rest of the characters was documented from random sample of five plants in each plot. The recommended agronomic practices and crop protection measures were followed during the crop growth period. Biometrical methods were followed to estimate genotypic and phenotypic coefficient of variation (Burton 1952), heritability in broad sense (Lush 1940), genetic advance (Johnson et al., 1955) and 
correlation and path coefficient analysis (Singh and Chaudhary, 1979).

\section{Results and Discussion}

The analysis of variance revealed significant differences among the genotypes for all the characters under study (Table 1). Thus, it indicated considerable amount of genetic variability among fifty chickpea germplasm.

\section{Estimation of genetic parameters}

Estimation of genetic parameters, correlation and path analysis helps to explore important characters during the selection for improving yield of chickpea. Genotypic variances, phenotypic variances, heritability, genotypic coefficient of variation (GCV), phenotypic coefficient of variation (PCV), genetic advance (GA) and genetic advance as percentage of mean, GA (\%) for all the yield contributing traits are shown in Table 2. PCV was higher than the corresponding GCV for all the traits indicating that there was an influence of the environment. Highest PCV and GCV were observed for effective pods per plant $(36.41 \%$ and $34.38 \%)$ followed by total number of pods per plant $(34.85 \%$ and $32.70 \%)$ and seed index (29.98\% and $29.78 \%)$ in Table 2. High GCV for number of pods per plant and 100-seed weight were also earlier reported by Jeena et al., (2005), Younis et al., (2008), Alwani et al., (2010) and Babbar et al.,(2012). Similar results were reported by Arora and Jeena (2001), Kumar et al., (2001), Jeena et al., (2005), Meena et al., (2006), Alwani et al., (2010) and Shreelakhsmi et al., (2010).

The high estimates of PCV and GCV for these traits suggested the possibility of yield improvement through selection of these traits. The traits studied showed moderate to high heritability ranging from $45.39 \%$ to $98.61 \%$. Among the traits studied the highest heritability was recorded by seed index $(98.61 \%)$ followed by harvest index $(90.81 \%)$, effective pods per plant $(89.14 \%)$, total number of pods per plant (88.01\%), secondary branches per plant (84.42\%), primary branches per plant $(84.31 \%)$ and plant height $(81.03 \%)$ (Table 2). The high heritability values of the considered traits in the present study indicated that those were less influenced by the environment and thus help in effective selection of the traits based on the phenotypic expression by adopting simple selection method and suggested the scope of genetic improvement. Similar results were also reported by Bicer and Sarkar (2008) and Younis et al., (2008).

The information on the magnitude of the inheritance of characters from parents to offspring is provided from heritability estimation, while genetic advance is helpful in finding out the actual gain expected under selection. In the present study, genetic advance as percent of mean estimated the highest for the effective pods per plant (66.86) followed by total number of pods per plant (63.19), seed index (60.94), primary branches per plant (39.38) and secondary branches per plant (32.96) among the yield contributing traits (Table 2). High heritability along with high genetic advance was observed for the traits, viz., effective pods per plant, total number of pods per plant, seed index, primary branches per plant and secondary branches per plant indicated that the characters were mostly governed by additive gene effects (Panse and Sukhatme, 1967) (Table 2).

So direct selection of these characters based on phenotypic expression by simple selection method would be effective due to accumulation of more additive genes leading to further improvement. The present findings are in support with Sharma and Saini (2010) and Sidramappa et al., 2010 and Mohan and Thiyagarajan (2019) 
Estimation of correlation coefficient among the traits

Relationships among yield and yield contributing traits were studied through analysis of correlation among them. Phenotypic and genotypic correlation coefficients among the studied traits of 50 chickpea germplasm are presented in Table 3. Correlation analysis among the yield and its contributing characters revealed that the genotypic correlation coefficients in most cases were higher than their phenotypic correlation coefficients indicating the association was largely due to genetic reason (Bhattacharyya et al., 2007). The phenotypic correlation coefficients in some cases were higher than their genotypic correlation, which indicates the suppressing effect of the environment that can alter the expression of characters at the phenotypic level. Grain yield per plant showed positive significant genotypic and phenotypic correlations with biological yield/plant $\left(0.684 * *, 0.775^{*}\right)$, seed index $\left(0.393^{* *}, 0.316^{* *}\right)$, harvest index $\left(0.363^{* *}, \quad 0.223^{* *}\right), \quad$ effective pod/plant $\left(0.299^{* *}, 0.368^{* *}\right)$, total pods/plant $\left(0.270^{* *}, 0.351^{* *}\right)$ and secondary branches per plant $\left(0.239^{* *}, \quad 0.197^{*}\right)$ whereas significant negative correlations with days to $50 \%$ flowering $\left(-0.289^{* *}, \quad-0,175^{*}\right)$ respectively. Days to $50 \%$ flowering exhibited positive significant genotypic and phenotypic correlations with days to maturity $\left(0.815^{* *}\right.$, $\left.0.566^{* *}\right)$, canopy temperature at pod filling stage $(0.433 * *, 0.353 * *)$, plant height $\left(0.289^{* *}, 0.251^{* *}\right)$ and canopy temperature at vegetative stage $(0.291 * *$ at genotypic level) and significant negative correlation with biological yield/plant $\left(-0.328^{* *},-0.178^{* *}\right)$, effective pods/plant (-0.196* at genotypic) and total pods/plant $\left(-0.179^{*}\right.$ at genotypic level) respectively. Days to maturity registered significant and positive association with secondary branches/plant $(0.272 * *, 0.194 *)$ and plant height $\left(0.238^{* *}, 0.175^{*}\right)$ at both genotypic and phenotypic level whereas chlorophyll index $(0.180 *)$ at genotypic level only. Chlorophyll index showed positive and significant correlations with secondary branches/plant $\left(0.317^{* *}, 0.205^{*}\right)$ at both genotypic and phenotypic level, but harvest index $\left(0.186^{*}\right)$ at genotypic level only whereas significant negative correlations with canopy temperature at pod filling stage ($\left.0.169^{*}\right)$ at genotypic level. Canopy temperature at vegetative stage exhibited positive significant genotypic and phenotypic correlations with canopy temperature at pod filling stage $\left(0.470^{* *}, 0.272 * *\right)$ respectively. Canopy temperature at pod filling stage and plant height showed non-significant association with all the characters. Number of primary branches/plant exhibited positive significant genotypic and phenotypic association with secondary branches/plant $\left(0.396^{* *}, 0.472^{* *}\right)$ and seed index $\left(0.248^{* *}\right.$, $0.227 * *)$ whereas it had significant and negative association with effective pods/plant $\left(-0.229^{*}\right)$ and total pod/plant $\left(-0.228^{* *}\right)$ at genotypic level only. Number of secondary branches/plant was significant positive genotypic and phenotypic association with total number of pod/plant $\left(0.176^{*}, 0.195^{*}\right)$ respectively. Total pod/plant recorded positive significant genotypic and phenotypic correlations with effective pods/plant $\left(0.978^{* *}, \quad 0.980^{* *}\right) \quad$ whereas biological yield/plant $\left(0.259^{* *}\right)$ showed positive significant correlation only at phenotypic level. Effective pods/plant had positive significant genotypic and phenotypic correlations with biological yield/plant $\left(0.174^{*}, 0.282^{* *}\right)$ respectively. Positive and significant association of biological yield/plant was observed with seed index (0.328**, $0.249 * *)$ whereas significant and negative correlation with harvest index $\left(0.426^{* *}\right.$, $0.435^{* *}$ ) both at genotypic and phenotypic level. Harvest index showed significant positive genotypic and phenotypic association with grain yield/plant $\left(0.363^{* *}, 0.223^{*}\right)$ 
respectively. Seed index exhibited positive significant genotypic and phenotypic correlations with grain yield per plant $\left(0.393^{* *}, 0.316^{* *}\right)$, biological yield per plant $\left(0.328^{* *}, 0.249^{* *}\right)$ and primary branches per plant $\left(0.248^{* *}, 0.227 * *\right)$ respectively.

Correlation analysis revealed that grain yield per plant showed significant and positive correlations with biological yield/plant, seed index, harvest index, effective pod/plant, total pods/plant and secondary branches per plant whereas it showed significant negative correlations with days to $50 \%$ flowering at both genotypic and phenotypic levels respectively (Table 3 ). Similar finding were reported by Talebi et al., (2007), Bicer and Sarkar (2008), Hahid et al., (2010), Ali et al., (2011), Babbar et al., (2012) and Kumar et al., (2017), Samyukta et al., (2017) and Agarwal et al., (2018).

\section{Estimation of path coefficient}

The results of the path coefficient analysis for phenotypic and genotypic correlations are shown in Table 4. Days to 50\% flowering had negative direct effect $(-0.026)$ at genotypic level and positive direct effect (0.017) on grain yield / plant at phenotypic level. It exhibited positive indirect effect via biological yield, total pods per plant, effective pods/plant, seed index, primary branches/plant at genotypic level and days to maturity, canopy temperature at pod filling stage, plant height, canopy temperature at vegetative stage, chlorophyll index, secondary branches/plant at phenotypic level only whereas negative indirect effects were exhibited by harvest index at genotypic and phenotypic level respectively, and days to maturity, chlorophyll index, canopy temperature at vegetative stage, canopy temperature at pod filling stage, plant height, secondary branches/plant at genotypic level and primary branches/plant, total pods/plant, effective pods/plants, biological yield and seed index at only phenotypic level.

Days to maturity had positive direct effect $(0.059,0.020)$ on grain yield per plant. It exhibited positive indirect effect via days to $50 \%$ flowering, chlorophyll index, canopy temperature at vegetative stage, canopy temperature at pod filling stage, plant and secondary branches per plant while it had negative indirect through, total no. of pods/plant, effective pods/plant, biological yield and harvest index. Chlorophyll index recorded positive direct effect $(0.038,0.006)$ on grain yield per plant. It exhibited positive indirect effect through days to maturity, harvest index, seed index, secondary branches/plant, days to $50 \%$ flowering, Primary branches/plant, total pods/plant, canopy temperature at vegetative stage. Direct effect at canopy temperature at vegetative stage on grain yield was positive $(0.025$, $0.004)$. It exhibited positive indirect effect via days to $50 \%$ flowering, canopy temperature at pod filling stage, days to maturity and chlorophyll index. Direct effect of canopy temperature at pod filling stage on grain yield per plant was positive $(0.008,0.007)$.

It exhibited positive indirect effect via, days to $50 \%$ flowering, Canopy temperature at vegetative stage, days to maturity, secondary branches/plant and effective pods/plant. Direct effect of plant height on grain yield/plant was positive $(0.033,0.013)$. It exhibited positive indirect effect through days to 50\% flowering, days to maturity, total pods/plant and effective pods/plant. Number of primary branches per plant had negative direct effect $(-0.007$, $0.009)$ on grain yield/plant. Positive indirect effect were also observed on total pods/plant, effective pods/plant, harvest index, days to $50 \%$ flowering, days to maturity, canopy temperature at pod filling stage and plant height. 
Table.1 Analysis of variance for fourteen characters in fifty chickpea germplasm

\begin{tabular}{|c|c|c|c|c|}
\hline $\begin{array}{l}\text { SI. } \\
\text { NO. }\end{array}$ & Characters & $\begin{array}{l}\text { Mean Sum of Squares } \\
\text { Replication (d. f.=2) }\end{array}$ & $\begin{array}{l}\text { Treatment } \\
\text { (d .f.=49) }\end{array}$ & $\begin{array}{c}\text { Error } \\
\text { (d. f. }=98)\end{array}$ \\
\hline 1. & Days to $50 \%$ flowering & 19.23 & $76.23 * *$ & 6.18 \\
\hline 2. & Days to maturity & 9.15 & $29.56^{* *}$ & 4.45 \\
\hline 3. & Chlorophyll index & 5.55 & $40.88 * *$ & 11.70 \\
\hline 4. & $\begin{array}{c}\text { Canopy Temperature at Vegetative } \\
\text { stage }\end{array}$ & 0.03 & $6.19 * *$ & 1.25 \\
\hline 5. & $\begin{array}{l}\text { Canopy Temperature at pod filling } \\
\text { stage }\end{array}$ & 6.82 & $23.87 * *$ & 3.25 \\
\hline 6. & Plant height $(\mathrm{cm})$ & 4.16 & $150.62 * *$ & 10.90 \\
\hline 7. & Primary branches per plant & 0.02 & $0.45 * *$ & 0.03 \\
\hline 8. & Secondary branches per plant & 0.02 & $2.32 * *$ & 0.13 \\
\hline 9. & Total no. of pods per plant & 0.81 & $522.47 * *$ & 22.70 \\
\hline 10. & Effective pods per plant & 0.16 & $372.94 * *$ & 14.56 \\
\hline 11. & Biological yield per plant & 20.27 & $34.91 * *$ & 5.80 \\
\hline 12. & Harvest index & 0.15 & $39.50 * *$ & 1.29 \\
\hline 13. & Seed index & 10.73 & $135.66^{* *}$ & 0.64 \\
\hline 14. & Grain yield per plant & 2.32 & $3.64 * *$ & 0.50 \\
\hline
\end{tabular}

* and ** Significant at $5 \%$ and $1 \%$ levels of significance, respectively.

Table.2 Estimates of genetic parameters for fourteen quantitative characters in fifty germplasm of Chickpea

\begin{tabular}{|c|c|c|c|c|c|c|c|c|}
\hline $\begin{array}{l}\text { SI. } \\
\text { NO. }\end{array}$ & Characters & $\sigma^{2} g$ & $\sigma^{2} p$ & GCV & PCV & $\begin{array}{c}\mathbf{h}^{2} \\
\text { (bs) } \%\end{array}$ & GA & $\begin{array}{c}\text { GA as \% } \\
\text { of mean }\end{array}$ \\
\hline 1. & Days to $50 \%$ flowering & 23.35 & 29.53 & 6.58 & 7.39 & 79.10 & 8.85 & 12.05 \\
\hline 2. & Days to maturity & 8.37 & 12.81 & 2.37 & 2.94 & 65.34 & 4.82 & 3.95 \\
\hline 3. & Chlorophyll index & 9.73 & 21.42 & 4.96 & 7.36 & 45.39 & 4.33 & 6.88 \\
\hline 4. & $\begin{array}{c}\text { Canopy Temperature at } \\
\text { Vegetative stage }\end{array}$ & 1.65 & 2.90 & 5.89 & 7.81 & 56.90 & 1.99 & 9.15 \\
\hline 5. & $\begin{array}{c}\text { Canopy Temperature at } \\
\text { pod filling stage }\end{array}$ & 6.86 & 10.12 & 8.14 & 9.88 & 67.86 & 4.45 & 13.82 \\
\hline 6. & Plant height $(\mathrm{cm})$ & 46.57 & 57.47 & 12.98 & 14.42 & 81.03 & 12.65 & 24.08 \\
\hline 7. & $\begin{array}{l}\text { Primary branches per } \\
\text { plant }\end{array}$ & 0.14 & 0.17 & 20.81 & 22.67 & 84.31 & 0.71 & 39.38 \\
\hline 8. & $\begin{array}{l}\text { Secondary branches per } \\
\text { plant }\end{array}$ & 0.72 & 0.86 & 17.41 & 18.95 & 84.42 & 1.62 & 32.96 \\
\hline 9. & $\begin{array}{c}\text { Total no. of pods per } \\
\text { plant }\end{array}$ & 166.59 & 189.29 & 32.70 & 34.85 & 88.01 & 24.94 & 63.19 \\
\hline 10. & Effective pods per plant & 119.46 & 134.02 & 34.38 & 36.41 & 89.14 & 21.26 & 66.86 \\
\hline 11. & $\begin{array}{l}\text { Biological yield per } \\
\text { plant }\end{array}$ & 9.70 & 15.50 & 13.45 & 16.99 & 62.60 & 5.08 & 21.91 \\
\hline 12. & Harvest index & 12.74 & 14.03 & 10.23 & 10.74 & 90.81 & 7.01 & 20.09 \\
\hline 13. & Seed index & 45.01 & 45.64 & 29.78 & 29.98 & 98.61 & 13.72 & 60.91 \\
\hline 14. & Grain yield per plant & 1.05 & 1.54 & 12.77 & 15.49 & 67.91 & 1.74 & 21.68 \\
\hline
\end{tabular}

$\boldsymbol{\sigma}^{2} \mathbf{g}=$ Genotypic variances, $\boldsymbol{\sigma}^{2} \mathbf{p}=$ Phenotypic variances, $\mathbf{G C V}=$ Genotypic coefficient of variation, $\mathbf{P C V}=$ Phenotypic coefficient of variation, $\mathbf{h} \mathbf{2} \mathbf{b s}=$ heritability in broad sense and $\mathbf{G A}=$ genetic advance. 
Table.3 Genotypic and phenotypic correlation coefficient for yield and yield attributing traits in fifty chickpea germplasm

\begin{tabular}{|c|c|c|c|c|c|c|c|c|c|c|c|c|c|c|c|}
\hline Characters & & $\begin{array}{c}\text { Days to } \\
50 \% \\
\text { flow. }\end{array}$ & $\begin{array}{l}\text { Days to } \\
\text { maturity }\end{array}$ & $\begin{array}{c}\text { Chloro } \\
\text { phyll } \\
\text { index }\end{array}$ & $\begin{array}{c}\text { CT @ } \\
\text { VS }\end{array}$ & $\begin{array}{c}\text { CT @ } \\
\text { PFS }\end{array}$ & $\begin{array}{c}\text { Plant } \\
\text { height }\end{array}$ & $\begin{array}{c}\text { Primary } \\
\text { branches } \\
\text { plant }^{-1}\end{array}$ & $\begin{array}{c}\text { Secondar } \\
y \\
\text { branches } \\
\text { plant }^{-1}\end{array}$ & $\begin{array}{c}\text { Total no. } \\
\text { of Pods } \\
\text { plant }^{-1}\end{array}$ & $\begin{array}{c}\text { Effective } \\
\text { pods } \\
\text { plant }^{-1}\end{array}$ & $\begin{array}{c}\text { Biological } \\
\text { yield } \\
\text { plant }^{-1}\end{array}$ & $\begin{array}{c}\text { Harvest } \\
\text { Index }\end{array}$ & Seed Index & $\begin{array}{c}\text { Grain } \\
\text { yield } \\
\text { plant }^{-1}\end{array}$ \\
\hline \multirow{2}{*}{$\begin{array}{c}\text { Days to } 50 \% \\
\text { flowering }\end{array}$} & G & 1.000 & $0.815 * *$ & 0.120 & $0.291 * *$ & $0.443 * *$ & $0.289 * *$ & -0.057 & 0.080 & $-0.179 *$ & $-0.196^{*}$ & $-0.328 * *$ & 0.003 & -0.118 & $-0.289 * *$ \\
\hline & $\mathrm{P}$ & 1.000 & $0.566^{* *}$ & 0.103 & 0.158 & $0.353 * *$ & $0.251 * *$ & -0.028 & 0.077 & -0.139 & -0.155 & $-0.178^{*}$ & -0.026 & -0.109 & $-0.175^{*}$ \\
\hline \multirow{2}{*}{$\begin{array}{c}\text { Days to } \\
\text { maturity }\end{array}$} & $\mathrm{G}$ & & 1.000 & $0.180^{*}$ & 0.036 & 0.112 & $0.238 * *$ & -0.011 & $0.272 * *$ & -0.045 & -0.119 & -0.104 & -0.018 & -0.005 & -0.069 \\
\hline & $\mathrm{P}$ & & 1.000 & 0.108 & 0.035 & 0.043 & $0.175^{*}$ & -0.010 & $0.194 *$ & -0.036 & -0.090 & -0.058 & -0.006 & 0.004 & -0.029 \\
\hline \multirow{2}{*}{$\begin{array}{l}\text { Chlorophyll } \\
\text { index }\end{array}$} & $\mathrm{G}$ & & & 1.000 & 0.052 & $-0.169 *$ & -0.020 & 0.067 & $0.317 * *$ & 0.076 & -0.012 & -0.140 & $0.186^{*}$ & 0.157 & 0.046 \\
\hline & $\mathrm{P}$ & & & 1.000 & 0.004 & -0.089 & -0.001 & 0.020 & $0.205^{*}$ & 0.011 & -0.042 & -0.112 & 0.116 & 0.112 & -0.025 \\
\hline \multirow[t]{2}{*}{ CT @ VS } & G & & & & 1.000 & $0.470 * *$ & -0.054 & -0.007 & -0.032 & -0.023 & -0.033 & -0.121 & -0.006 & -0.025 & -0.106 \\
\hline & $\mathrm{P}$ & & & & 1.000 & $0.272 * *$ & -0.010 & 0.047 & 0.0361 & -0.044 & -0.049 & -0.142 & 0.013 & -0.008 & -0.134 \\
\hline \multirow[t]{2}{*}{ CT @ PFS } & $\mathrm{G}$ & & & & & 1.000 & -0.059 & -0.125 & 0.024 & -0.030 & 0.026 & -0.066 & -0.059 & -0.122 & -0.103 \\
\hline & $\mathrm{P}$ & & & & & 1.000 & -0.057 & -0.084 & 0.016 & 0.005 & 0.050 & 0.016 & -0.066 & -0.097 & -0.016 \\
\hline \multirow[t]{2}{*}{ Plant height } & $\mathrm{G}$ & & & & & & 1.000 & -0.117 & -0.078 & 0.047 & 0.029 & -0.109 & -0.022 & -0.111 & -0.094 \\
\hline & $\mathrm{P}$ & & & & & & 1.000 & -0.111 & -0.070 & 0.035 & 0.020 & -0.105 & -0.007 & -0.096 & -0.097 \\
\hline \multirow{2}{*}{$\begin{array}{l}\text { Pri. branches } \\
\text { plant }^{-}\end{array}$} & $\mathrm{G}$ & & & & & & & 1.000 & $0.396^{* *}$ & $-0.228 * *$ & $-0.229 * *$ & 0.068 & -0.095 & $0.248 * *$ & -0.011 \\
\hline & $\mathrm{P}$ & & & & & & & 1.000 & $0.472 * *$ & -0.154 & -0.156 & 0.090 & -0.125 & $0.227 * *$ & 0.007 \\
\hline \multirow{2}{*}{$\begin{array}{l}\text { Sec. branches } \\
\text { plant }^{-1}\end{array}$} & G & & & & & & & & 1.000 & $0.176^{*}$ & 0.098 & 0.135 & 0.094 & 0.133 & $0.239 * *$ \\
\hline & $\mathrm{P}$ & & & & & & & & 1.000 & $0.195^{*}$ & 0.128 & 0.141 & 0.035 & 0.123 & $0.197 *$ \\
\hline \multirow{2}{*}{$\begin{array}{l}\text { Total no. of } \\
\text { Pods plant }{ }^{-1}\end{array}$} & $\mathrm{G}$ & & & & & & & & & 1.000 & $0.978 * *$ & 0.133 & 0.149 & 0.001 & $0.270 * *$ \\
\hline & $\mathrm{P}$ & & & & & & & & & 1.000 & $0.980 * *$ & $0.259 * *$ & 0.082 & 0.001 & $0.351 * *$ \\
\hline \multirow{2}{*}{$\begin{array}{l}\text { Effective pods } \\
\text { plant }^{-1}\end{array}$} & G & & & & & & & & & & 1.000 & $0.174^{*}$ & 0.139 & -0.001 & $0.299 * *$ \\
\hline & $\mathrm{P}$ & & & & & & & & & & 1.000 & $0.282 * *$ & 0.077 & -0.001 & $0.368 * *$ \\
\hline \multirow{2}{*}{$\begin{array}{c}\text { Biological } \\
\text { yield plant }^{-1}\end{array}$} & $\mathrm{G}$ & & & & & & & & & & & 1.000 & $-0.426^{* *}$ & $0.328 * *$ & $0.684 * *$ \\
\hline & $\mathrm{P}$ & & & & & & & & & & & 1.000 & $-0.435 * *$ & $0.249 * *$ & $0.775 * *$ \\
\hline \multirow[t]{2}{*}{ Harvest Index } & $\mathrm{G}$ & & & & & & & & & & & & 1.000 & 0.061 & $0.363 * *$ \\
\hline & $\mathrm{P}$ & & & & & & & & & & & & 1.000 & 0.061 & $0.223 * *$ \\
\hline \multirow[t]{2}{*}{ Seed Index } & $\mathrm{G}$ & & & & & & & & & & & & & 1.000 & $0.393 * *$ \\
\hline & $\mathrm{P}$ & & & & & & & & & & & & & 1.000 & $0.316^{* *}$ \\
\hline
\end{tabular}

*, ** Significant at $5 \%$ and $1 \%$ levels of significance, respectively;

CT@VS=Canopy temperature at vegetative stage; CT @PFS= Canopy temperature at pod filling stage 
Table.4 Direct (diagonal) and indirect genotypic and phenotypic effects of different characters on grain yield in chickpea

\begin{tabular}{|c|c|c|c|c|c|c|c|c|c|c|c|c|c|c|c|}
\hline Characters & & $\begin{array}{c}\text { Days to } \\
\mathbf{5 0 \%} \\
\text { flowerin } \\
\mathbf{g} \\
\end{array}$ & $\begin{array}{l}\text { Days to } \\
\text { maturity }\end{array}$ & $\begin{array}{l}\text { Chloroph } \\
\text { yll index }\end{array}$ & CT @VS & $\begin{array}{l}\text { CT @ } \\
\text { PFS }\end{array}$ & $\begin{array}{l}\text { Plant } \\
\text { height }\end{array}$ & $\begin{array}{c}\text { primary } \\
\text { branches }^{-1} \text { plant }^{-1}\end{array}$ & $\begin{array}{c}\text { Secondary } \\
\text { branches }^{-1} \text { plant }^{-1}\end{array}$ & $\begin{array}{l}\text { Total no. } \\
\text { of Pods } \\
\text { plant }^{-1}\end{array}$ & $\begin{array}{c}\text { Effective } \\
\text { pods } \\
\text { plant }^{-1}\end{array}$ & $\begin{array}{c}\text { Biological } \\
\text { yield } \\
\text { plant }^{-1}\end{array}$ & $\begin{array}{c}\text { Harvest } \\
\text { Index }\end{array}$ & $\begin{array}{l}\text { Seed } \\
\text { Index }\end{array}$ & $\begin{array}{c}\text { Grain } \\
\text { yield } \\
\text { plant }^{-1}\end{array}$ \\
\hline \multirow{2}{*}{$\begin{array}{l}\text { Days to } 50 \% \\
\text { flowering }\end{array}$} & G & $-\mathbf{- 0 . 0 2 6}$ & -0.021 & -0.003 & -0.007 & -0.011 & -0.007 & 0.002 & -0.002 & 0.005 & 0.005 & 0.008 & -0.0001 & 0.003 & -0.289 \\
\hline & $\mathrm{P}$ & 0.017 & 0.010 & 0.002 & 0.003 & 0.006 & 0.004 & -0.0005 & 0.001 & -0.002 & -0.003 & -0.003 & -0.0004 & -0.002 & -0.175 \\
\hline \multirow[t]{2}{*}{ Days to maturity } & G & 0.048 & 0.059 & 0.011 & 0.002 & 0.007 & 0.014 & -0.001 & 0.016 & -0.003 & -0.007 & -0.006 & -0.001 & -0.0003 & -0.069 \\
\hline & $\mathrm{P}$ & 0.011 & 0.020 & 0.002 & 0.001 & 0.001 & 0.004 & -0.0002 & 0.004 & -0.001 & -0.002 & -0.001 & -0.0001 & 0.0001 & -0.029 \\
\hline \multirow{2}{*}{$\begin{array}{l}\text { Chlorophyll } \\
\text { index }\end{array}$} & G & 0.005 & 0.007 & 0.038 & 0.002 & -0.007 & -0.001 & 0.003 & 0.012 & 0.003 & -0.0005 & -0.005 & 0.007 & 0.006 & 0.046 \\
\hline & $\mathrm{P}$ & 0.001 & 0.001 & 0.006 & 0.000 & -0.0005 & 0.000 & 0.0001 & 0.001 & 0.0001 & -0.0003 & -0.001 & 0.001 & 0.001 & -0.025 \\
\hline \multirow[t]{2}{*}{ CT @ VS } & G & 0.007 & 0.001 & 0.001 & 0.025 & 0.012 & -0.001 & -0.0002 & -0.001 & -0.001 & -0.001 & -0.003 & -0.0001 & -0.001 & -0.107 \\
\hline & $\mathrm{P}$ & 0.001 & 0.0001 & 0.000 & 0.004 & 0.001 & 0.000 & 0.0002 & 0.0002 & -0.0002 & -0.0002 & -0.001 & 0.0001 & 0.000 & -0.134 \\
\hline \multirow[t]{2}{*}{ CT @ PFS } & G & 0.004 & 0.001 & -0.001 & 0.004 & 0.008 & -0.0005 & -0.001 & 0.0002 & -0.0002 & 0.0002 & -0.0005 & -0.0005 & -0.001 & -0.103 \\
\hline & $\mathrm{P}$ & 0.003 & 0.0003 & -0.001 & 0.002 & 0.007 & -0.0004 & -0.001 & 0.0001 & 0.000 & 0.0003 & 0.0001 & -0.0005 & -0.001 & -0.016 \\
\hline \multirow[t]{2}{*}{ Plant height } & G & 0.010 & 0.008 & -0.001 & -0.002 & -0.002 & 0.033 & -0.004 & -0.003 & 0.002 & 0.001 & -0.004 & -0.001 & -0.004 & -0.094 \\
\hline & $\mathrm{P}$ & 0.003 & 0.002 & 0.000 & -0.0001 & -0.001 & 0.013 & -0.001 & -0.001 & 0.0004 & 0.0003 & -0.001 & -0.0001 & -0.001 & -0.097 \\
\hline \multirow{2}{*}{$\begin{array}{l}\text { Pri. branches } \\
\text { plant }\end{array}$} & G & 0.0004 & 0.0001 & -0.0005 & 0.000 & 0.001 & 0.001 & -0.007 & -0.003 & 0.002 & 0.002 & -0.0005 & 0.001 & -0.002 & -0.011 \\
\hline & $\mathrm{P}$ & 0.0002 & 0.0001 & -0.0002 & -0.0004 & 0.001 & 0.001 & -0.009 & -0.004 & 0.001 & 0.001 & -0.001 & 0.001 & -0.002 & 0.007 \\
\hline \multirow{2}{*}{$\begin{array}{l}\text { Sec. branches } \\
\text { plant }^{-1}\end{array}$} & $\mathrm{G}$ & 0.001 & 0.003 & 0.003 & -0.0003 & 0.0002 & -0.001 & 0.004 & 0.010 & 0.002 & 0.001 & 0.001 & 0.001 & 0.001 & 0.239 \\
\hline & $\mathrm{P}$ & 0.001 & 0.002 & 0.003 & 0.0005 & 0.0002 & -0.001 & 0.006 & 0.013 & 0.003 & 0.002 & 0.002 & 0.0004 & 0.002 & 0.197 \\
\hline \multirow{2}{*}{$\begin{array}{l}\text { Total no. of Pods } \\
\text { plant }^{-1}\end{array}$} & G & 0.012 & 0.003 & -0.005 & 0.002 & 0.002 & -0.003 & 0.015 & -0.012 & -0.068 & -0.066 & -0.009 & -0.010 & -0.0001 & 0.270 \\
\hline & $\mathrm{P}$ & -0.009 & -0.002 & 0.001 & -0.003 & 0.0003 & 0.002 & -0.009 & 0.012 & 0.061 & 0.060 & 0.016 & 0.005 & 0.000 & 0.351 \\
\hline \multirow{2}{*}{$\begin{array}{l}\text { Effective pods } \\
\text { plant }^{-1}\end{array}$} & G & -0.015 & -0.009 & -0.001 & -0.003 & 0.002 & 0.002 & -0.017 & 0.007 & 0.074 & 0.076 & 0.013 & 0.011 & 0.000 & 0.299 \\
\hline & $\mathrm{P}$ & 0.007 & 0.004 & 0.002 & 0.002 & -0.002 & -0.001 & 0.007 & -0.006 & -0.045 & -0.046 & -0.013 & -0.004 & 0.000 & 0.368 \\
\hline \multirow{2}{*}{$\begin{array}{l}\text { Biological yield } \\
\text { plant }^{-1}\end{array}$} & G & -0.337 & -0.107 & -0.144 & -0.124 & -0.068 & -0.112 & 0.070 & 0.139 & 0.137 & 0.179 & 1.026 & -0.437 & 0.337 & 0.684 \\
\hline & $\mathrm{P}$ & -0.191 & -0.063 & -0.121 & -0.152 & 0.018 & -0.113 & 0.097 & 0.152 & 0.278 & 0.302 & 1.073 & -0.466 & 0.268 & 0.775 \\
\hline \multirow[t]{2}{*}{ Harvest Index } & G & 0.003 & -0.014 & 0.147 & -0.004 & -0.047 & -0.018 & -0.076 & 0.074 & 0.118 & 0.111 & -0.338 & 0.793 & 0.048 & 0.363 \\
\hline & $\mathrm{P}$ & -0.018 & -0.004 & 0.080 & 0.009 & -0.045 & -0.005 & -0.085 & 0.024 & 0.056 & 0.053 & -0.298 & 0.685 & 0.042 & 0.223 \\
\hline \multirow[t]{2}{*}{ Seed Index } & G & -0.001 & 0.000 & 0.001 & -0.0001 & -0.001 & -0.0005 & 0.001 & 0.001 & 0.000 & 0.000 & 0.002 & 0.0003 & 0.005 & 0.393 \\
\hline & $\mathrm{P}$ & -0.001 & 0.000 & 0.001 & -0.0001 & -0.001 & -0.001 & 0.002 & 0.001 & 0.000 & 0.000 & 0.003 & 0.001 & 0.010 & 0.316 \\
\hline $\mathbf{P}$ & & $\begin{array}{l}\mathrm{URE}= \\
\mathrm{URE}=\end{array}$ & $\begin{array}{l}0.9964 \\
0.9886\end{array}$ & $\begin{array}{l}\text { SIDUAL } \\
\text { SIDUAL } \mathrm{F}\end{array}$ & $\begin{array}{l}\text { FFECT }= \\
\text { FECT }=\end{array}$ & $\begin{array}{l}0.0601) \\
0.1070)\end{array}$ & r@PFS & & & $\begin{array}{l}\text { ve stage } \\
\text { ing stag }\end{array}$ & & & & & \\
\hline
\end{tabular}


Fig.1 Histogram showing estimates of Genetic Parameters for fourteen quantitative characters in chickpea

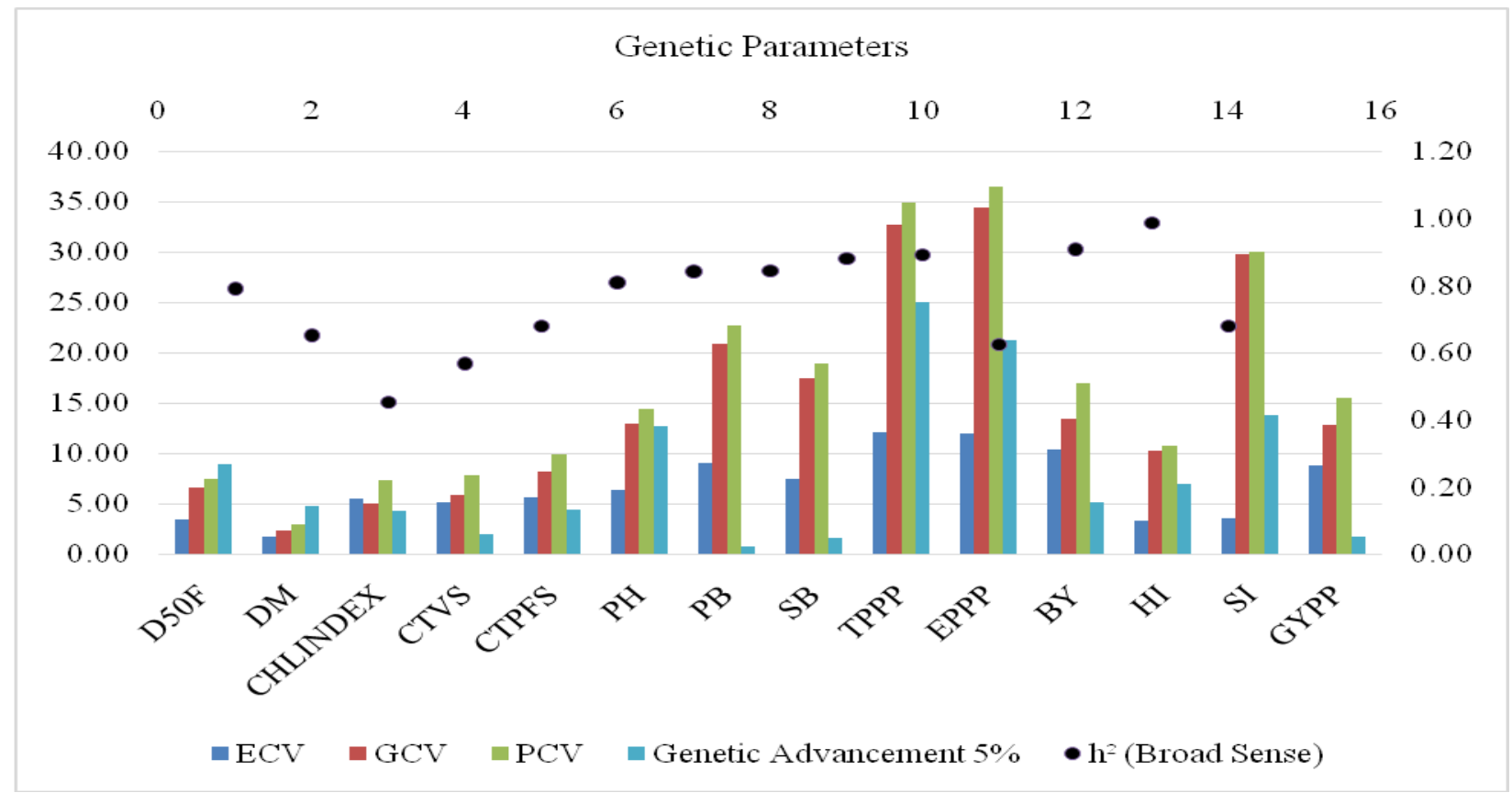

Fig.2 Phenotypic path diagram for grain yield per plant

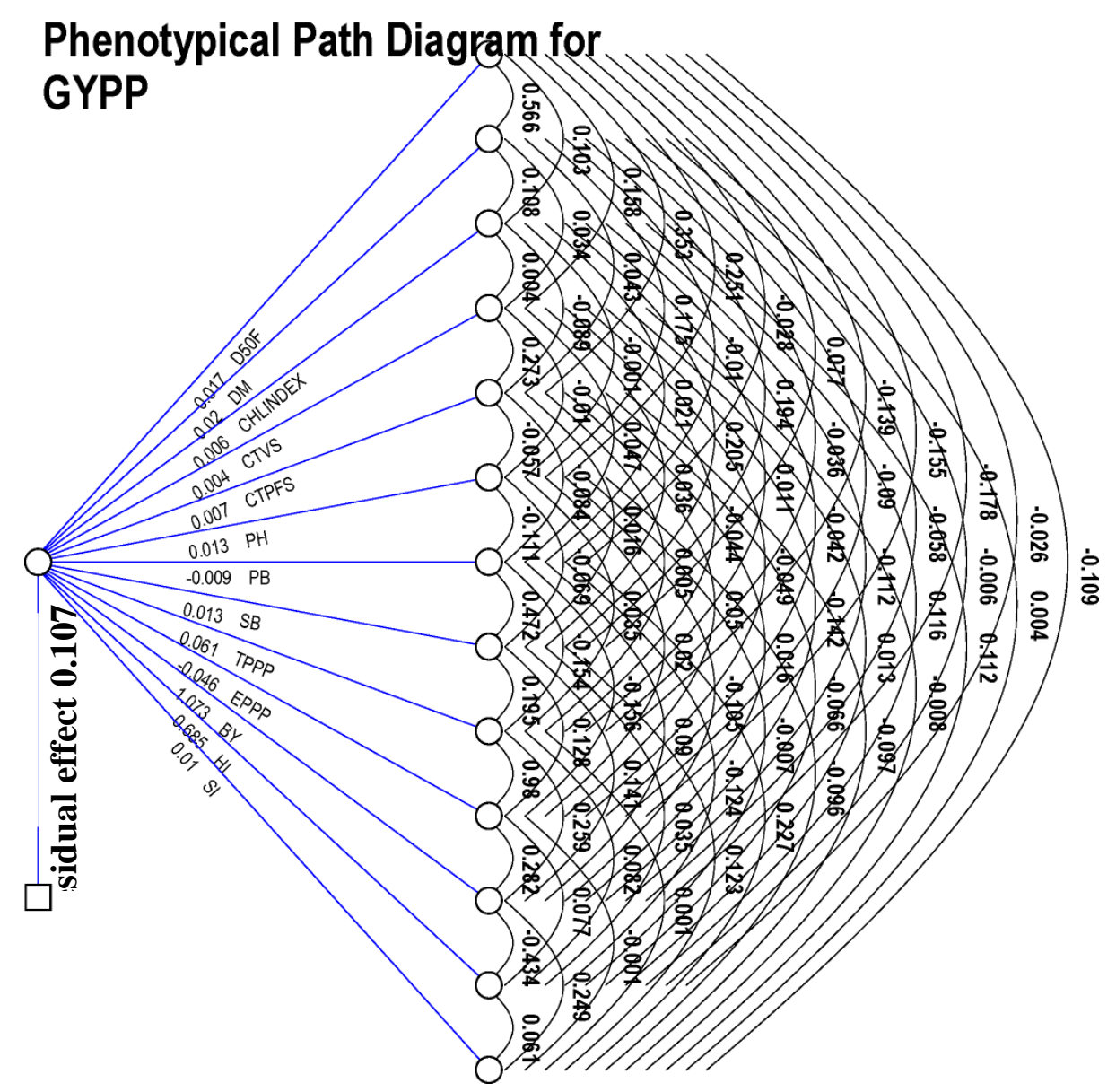


Number of secondary branches per plant had positive direct effect $(0.010,0.013)$ on grain yield per plant. It exhibited positive indirect effect through days to $50 \%$ flowering, days to maturity, chlorophyll index, canopy temperature at pod filling stage, primary branches/plant, total no. of pods/plant, effective pods/plant, biological yield/plant, harvest index and 100-seed weight. But its indirect effect was negative via plant height.

Total number of pods per plant showed negative direct effect (-0.068) at genotypic level whereas positive effect $(0.061)$ at phenotypic level on grain yield per plant. Positive indirect effect was also observed on canopy temperature at pod filling stage at genotypic and phenotypic level, whereas days to $50 \%$ flowering, days to maturity, canopy temperature at vegetative stage, canopy temperature at pod filling stage, Primary branches/plant at genotypic level. Effective pods per plant showed positive direct effect (0.076) at genotypic level where as negative effect (-0.046) at phenotypic level on grain yield/plant. It exhibited positive indirect effect on canopy temperature at pod filling stage, plant height, secondary branches/plant, total pods/plant, biological yield/plant, harvest index at genotypic level and days to $50 \%$ flowering, days to maturity, chlorophyll index, canopy temperature at vegetative stage, primary branches/plant at phenotypic level.

Direct effect of biological yield per plant on grain yield/plant was positive $(1.026,1.073)$. Its indirect effect via, seed index, effective pods/plant, secondary branches/plant, total pods/plant and primary branches/plant were positive. Harvest index showed positive direct effect $(0.793,0.685)$ on grain yield per plant. It had positive indirect effect via chlorophyll index, effective pods/plant, total pods/plant, secondary branches/plant and seed index at both genotypic and phenotypic level but days to $50 \%$ flowering at genotypic level only.
Seed index exhibited positive direct effect $(0.005,0.010)$ on grain yield/plant. It had positive indirect effect via biological yield/plant, primary branches/plant, secondary branches/plant, chlorophyll index and harvest index.

At the both phenotypic and genotypic levels, biological yield per plant, harvest index, days to maturity, chlorophyll index, seed index, plant height and secondary branches per plant had direct positive effect on yield per plant had given the highest contribution on yield per plant (Table 4). The earlier studies for direct effect on grain yield for biological yield and harvest index were reported by Babbar and Patel (2005), Kuldeep et al., (2014), Naveed et al., (2012) and Tadesse et al., (2016) and Kumar et al., (2017) whereas, Talebi et al., (2007), found positive indirect effect on seed yield.

Estimation of genetic parameters, correlation analysis and path coefficient analysis revealed that the biological yield/plant, seed index, harvest index, effective pod/plant, total number of pods/plant and secondary branches per plant were the most reliable traits for yield improvement in chickpea. So the utmost importance should be given to these characters during the selection for yield improvement in chickpea.

\section{References}

Agrawal, T., Kumar, A., Kumar, S., Kumar, A., Kumar, R.R., Kumar, S. and Singh, P.K. (2018). Correlation and Path Coefficient Analysis for Grain Yield and yield Components in Chickpea (Cicer arietinum L.) under Normal and Late Conditions of Bihar, India. Int. J. Curr. Microbiol. App. Sci., 7(2): 16331642.

Arora, P.P. and Jeena, A.S. (2001). Genetic variability studies in chickpea. Legume 
Research-An International Journal. 24(2):137-138.

Ali, Q., Ahsan, M., Khaliq, I., Elahi, M., Shahbaz, M., Ahmed, W. and Naees, M. (2011). Estimation of genetic association of yield and quality traits in chickpea (Cicer arietinum L.). International Research Journal of Plant Science, 2(6):166-169.

Alwani, H., Moulla, M. and Chouhan, W. (2010). Genotype environment interaction and genetic parameter in Chickpea (Cicer arietinum L.) Landraces. Journal of Agricultural Science. 2(1): 153-157.

Babbar, A. and Patel, S.K. (2005). Correlation and Path analysis in desi chickpea under Kymore plateau zone of Madhya Pradesh. JNKVV Research Journal, 39(1): 47-51.

Babbar, A., Prakash, V., Prakash,T. and Iquabal, M.A. (2012). Genetic variability of chickpea (Cicer arietinum L.) under late sown condition. Legume Research. 35(1): 17.

Bhattacharya, A. and Ganguly, S.B. (2007). Genetic diversity in chickpea under normal and late sown codition. Legume Research, 21(3): 171-176.

Bicer, B.T. and Sakar, D. (2008). Heritability and path analysis of some economical characteristics in lentil. Journal of Central Europe Agriculture. 9(1): 191196.

Burton, G.W. (1952). Quantitative inheritance of grasses. Proceeding $6^{\text {th }}$ Int.Grassland congress. 1: 277-283.

Hahid, S., Malik, R., Bakhsh, A., Asif, M.A., Iqbal, U. and Iqbal, S.M. (2010). Assessment of genetic variability and interrelationship among some agronomic traits in chickpea. International Journal of Agricultural Biology. 12(1): 81-85.

Jeena, A.S., Arora, P.S. and Ojha, O.P. (2005).
Variability and correlation studies for yield and its components in chickpea. Legume Research. 28(2):146-148.

Johnson, H.W., Robinson, H.F. and Comstock, R.E. (1955). Genotypic and Phenotypic correlation in soybean and their implications in selection. Agron. J. 47: 477-482.

Kuldeep, R.K., Pandey, S., Babbar, A. and Mishra, D.K. (2014). Genetic variability, character association and path coefficient analysis in chickpea grown under heat stress condition. Electronic Journal of Plant Breeding. 5(4):812-819.

Kumar, S., Kumar, A., Kumar, A., Kumar, R.R., Roy, R.K. and Agrawal, T. (2017). Genetic Variability of Chickpea Genotypes under Heat Stress Condition: Character Association and Path Coefficient Based Analysis. Indian Journal of Ecology. 44 (Special Issue-4): 59-64.

Lush, J. L. (1940). Intra-sire correlation and regression of offspring in rams as a method of estimating heritability of characters. Proceedings of the America Philosophical Society on animal Product. 33: 292-301.

Naveed, M., Shafiq, M., Rafiq, C.M. and Zahid, M.A. (2015). Genetic diversity in new chickpea accessions for fusarium wilt resistance, canopy temperature and yield components under drought milieus. Australian Journal of Crop science. 9(6):538-544.

Panse V.G. and Sukhatme P.V. (1967). Statistical method for agricultural workers, ICAR, New Delhi, 2(1): 381384.

Samyuktha, S.M., Kannan B.J.R. and Geethanjali, S. (2018). Molecular genetic diversity and population structure analysis in chickpea germplasm using SSR Markers. Int. J. Curr. Microbiol. App. Sci., 7(2): 639- 
651.

Sidramappa, S.A. Patil and Salimath, P.M. (2010). Pattern of diversity based on phenplogical and productivity traits in chickpea (Cicer arietinum L.). Electronic Journal of Plant Breeding, 1(16): 1380-1385.

Talebi, R., Fayaz, F. and Jelodar, N.A.B. (2007). Correlation and path coefficient analysis of yield and yield components of chickpea (Cicer arietinum L.) under dry land condition in the west of Iran. Asian Journal of Plant Science. 6(7):1151-1154.
Tadesse M, Fikre A, Eshete M, Girman N, Korbun L, Mohamed R, Bekele D, Funga A and Ojiewo, C.O. (2016). Correlation and path coefficient analysis for various quantitative traits in desi chickpea genotypes under rainfed conditions in Ethiopia. J. of Agri. Sci., 8(12):112.

Younis, N., Hanif, M., Sadiq, S., Abbas, G., Asghar, M.J. and Haq, M.A. 2008. Estimates of genetic parameters and path analysis in lentil (Lens culinaris Medik). Pakistan Journal of Agricultural Science. 45(3): 44-48.

\section{How to cite this article:}

Sanjay Kumar, B. G. Suresh, Anand Kumar and Lavanya, G. R. 2019. Genetic Variability, Correlation and Path Coefficient Analysis in Chickpea (Cicer arietinum L.) for Yield and its Component Traits. Int.J.Curr.Microbiol.App.Sci. 8(12): 2341-2352.

doi: https://doi.org/10.20546/ijcmas.2019.812.276 